\title{
Research Communication Olopatadine Suppresses the Migration of THP-1 Monocytes Induced by S100A12 Protein
}

\author{
Kazuya Kishimoto, Satoshi Kaneko, Kenji Ohmori, Tadafumi Tamura, and Kazuhide Hasegawa
}

Pharmaceutical Research Center, Kyowa Hakko Kogyo Co, Ltd, 1188 Shimotogari, Nagaizumi-cho, Sunto-gun, Shizuoka 411-8731, Japan

Received 13 September 2005; Accepted 13 October 2005

Olopatadine hydrochloride (olopatadine) is an antiallergic drug with histamine $\mathrm{H}_{1}$ receptor antagonistic activity. Recently, olopatadine has been shown to bind to S100A12 which is a member of the S100 family of calcium-binding proteins, and exerts multiple proinflammatory activities including chemotaxis for monocytes and neutrophils. In this study, we examined the possibility that the interaction of olopatadine with S100A12 inhibits the proinflammatory effects of S100A12. Pretreatment of olopatadine with S100A12 reduced migration of THP-1, a monocyte cell line, induced by S100A12 alone, but did not affect recombinant human regulated upon activation, normal T cell expressed and secreted (RANTES)-induced migration. Amlexanox, which also binds to S100A12, inhibited the THP-1 migration induced by S100A12. However, ketotifen, another histamine $\mathrm{H}_{1}$ receptor antagonist, had little effect on the activity of S100A12. These results suggest that olopatadine has a new mechanism of action, that is, suppression of the function of S100A12, in addition to histamine $\mathrm{H}_{1}$ receptor antagonistic activity.

Copyright (c) 2006 Kazuya Kishimoto et al. This is an open access article distributed under the Creative Commons Attribution License, which permits unrestricted use, distribution, and reproduction in any medium, provided the original work is properly cited.

\section{INTRODUCTION}

S100A12 belongs to the S100 family of EF-hand-type calcium-binding proteins [1]. These proteins are involved in the regulation of a variety of intracellular activities, including protein phosphorylation, enzymatic activities, $\mathrm{Ca}^{2+}$ homeostasis, and intermediate filament polymerization [2]. On the other hand, several S100 proteins including S100A12 are secreted and have extracellular activities [2]. S100A12 belongs to a subset of the $\mathrm{S} 100$ protein family, which is termed the myeloid-related proteins (MRPs) [3-5], because expression of these proteins is almost completely restricted to cells of myeloid origin, such as neutrophils and monocytes. MRPs have proinflammatory effects in the extracellular milieu $[6,7]$. S100A12 is detected in the synovial fluid and plasma of patients with gout, rheumatoid arthritis, and psoriatic arthritis [8], suggesting that S100A12 may be involved in the inflammation in these diseases.

S100A12 from bovine lung extract was identified as a ligand for the receptor for advanced glycation end products (RAGE), which is expressed on macrophages, endothelium, and lymphocytes. Endothelial cells incubated with S100A12 exhibited increased expression of intercellular adhesion molecule-1 and vascular cell adhesion molecule-1 through the activation of nuclear factor- $\kappa$ B [9]. In addition,
S100A12 induced the expression of tumor necrosis factor$\alpha$ and interleukin- $1 \beta$ in a murine macrophage cell line [8]. Footpad injection of bovine S100A12 into mice resulted in the infiltration of leukocytes, and anti-S100A12 antibody blocked leukocyte recruitment in murine models of delayedtype hypersensitivity induced by methylated bovine serum albumin [9]. Furthermore, human recombinant S100A12 has been reported to be chemotactic for neutrophils and monocytes in vitro and in vivo $[10,11]$. Thus, S100A12 and RAGE are attractive targets for the treatment of inflammation.

It has been reported that anti-allergic drugs/mast cell stabilizers such as amlexanox and cromolyn specifically bind to S100A12 and S100A13 which are another member of the S100 protein family [12]. It has also been reported that amlexanox repressed the fibroblast growth factor 1 (FGF-1) release induced by S100A13 [13, 14]. Therefore, it is expected that binding of these drugs to S100A12 result in the inhibition of its function, which may contribute to antiallergic or anti-inflammatory effects of these drugs.

Olopatadine hydrochloride (olopatadine: (Z)-11-(3-Dimethylaminopropylidene)-6,11-dihydrodibenz [b,e] oxepin2 -acetic acid monohydrochloride, ALLELOCK ${ }^{\circledR}$, Kyowa Hakko Kogyo Co, Ltd, Japan) is an antiallergic agent with histamine $\mathrm{H}_{1}$ receptor antagonistic action that is indicated 
for the signs and symptoms of allergic rhinitis, chronic urticaria, eczema dermatitis, prurigo, pruritis cutaneous, psoriasis vulgaris, and erythema exsudativum multiform [15]. Olopatadine exhibits potent antihistamine activity in vivo following its systemic administration. In addition to its potent antihistaminic effect, previous studies have demonstrated that olopatadine inhibits the release of inflammatory lipid mediators such as leukotrienes and thromboxanes from human polymorphonuclear leukocytes and eosinophils [15]. Olopatadine also reduces the tachykinin release from peripheral sensory nerve endings [16]. Olopatadine inhibits eosinophil infiltration in both rat allergic rhinitis and mice chronic contact hypersensitivity models [17, 18]. However, the precise mechanism of inhibitory effects of olopatadine on infiltration of leukocytes is still unclear.

Recently, Okada et al have found that olopatadine binds specifically to S100A12 and S100A13 in a calcium-dependent manner as amlexanox and cromolyn do [19]. In the present study, we examined the inhibitory effect of olopatadine on the function of recombinant human S100A12.

\section{MATERIALS AND METHODS}

\section{Materials}

Olopatadine hydrochloride (olopatadine) was synthesized in Yokkaichi Plant, Kyowa Yuka Co, Ltd (Mie, Japan). Ketotifen fumarate (ketotifen) was purchased from Sigma Chemical (St Louis, Mo, USA). Amlexanox was extracted from SOLFA ${ }^{\circledR}$ tablets purchased from Takeda Chemical Industries Inc (Osaka, Japan). These drugs were dissolved in DMSO. Recombinant human regulated upon activation, normal $\mathrm{T}$ cell expressed and secreted (RANTES) was purchased from R\&D SYSTEMS (Minneapolis, Minn, USA).

\section{Recombinant S100A12 protein}

Human S100A12 cDNA was a gift from Dr Ryoji Kobayashi (Department of Signal Transduction Sciences, Kagawa University Faculty of Medicine, Kagawa, Japan). Recombinant protein was expressed in E coli strain BL21 (DE3) and purified as previously described by Yamashita et al [20], with some modifications. Briefly, $300 \mathrm{~mL}$ DE3 culture was grown at $37^{\circ} \mathrm{C}$ to an OD600 of $0.6-0.8$ and induced with $1 \mathrm{mmol} / \mathrm{L}$ isopropyl- $\beta$-D-thiogalactopyranoside for $4 \mathrm{~h}$ at $37^{\circ} \mathrm{C}$. The cell pellet was resuspended in hypotonic buffer, $10 \mathrm{mmol} / \mathrm{L}$ Tris- $\mathrm{HCl}$ ( $\mathrm{pH} 7.5$ ) containing $1 \mathrm{mmol} / \mathrm{L}$ ethylenediaminetetraacetic acid, and then centrifuged at $10000 \mathrm{~g}$ for $60 \mathrm{~min}$. The supernatant was dialyzed against $20 \mathrm{mmol} / \mathrm{L}$ Tris- $\mathrm{HCl}$ (pH 7.5) (buffer A), and loaded onto a HiPrep 16/10 Q XL column (Amersham Biosciences, Piscataway, NJ, USA) equilibrated with buffer A. The protein was eluted with a linear gradient from 0 to $250 \mathrm{mmol} / \mathrm{L} \mathrm{NaCl}$. Fractions containing the protein were pooled and loaded onto a HiLoad 16/60 Superdex 75 pg (Amersham Biosciences) equilibrated with buffer A. Fractions containing the protein were pooled and further purified using a Mono Q HR 5/5 column (Amersham Biosciences) equilibrated with buffer A. The protein was eluted with a linear gradient from 0 to $200 \mathrm{mmol} / \mathrm{L}$
$\mathrm{NaCl}$, and stored at $-80^{\circ} \mathrm{C}$. Purity was confirmed by sodium dodecyl sulfate-polyacrylamide gel electrophoresis. In S100A12 preparations used for this study, endotoxin levels were less than $0.1 \mathrm{pg} / \mu \mathrm{g}$ of S100A12 as measured by the limulus amebocyte assay (Bio Whittaker, Walkersville, Md, USA).

\section{Cell culture}

Human monocytic THP-1 cells (American Type Culture Collection, Manassas, Va, USA) were cultured in RPMI 1640 supplemented with $10 \mathrm{vol} \%$ heat-inactivated fetal bovine serum (FBS) (Cansera, Etobicoke, ON, Canada), $100 \mathrm{U} / \mathrm{mL}$ penicillin and $100 \mu \mathrm{g} / \mathrm{mL}$ streptomycin (Invitrogen, Grand Island, $\mathrm{NY}, \mathrm{USA}$ ) at $37^{\circ} \mathrm{C} 5 \% \mathrm{CO}_{2}$ in air.

\section{Cell migration assay}

The migration of THP-1 monocytes was studied using a Chemotaxicell 24-well disposable chamber with $5 \mu \mathrm{m}$ pores (Kurabo Industries Ltd, Osaka, Japan). Cultured cells were harvested and washed three times with Hank's balanced salt solution and then re-suspended in assay medium (RPMI 1640 with $0.5 \mathrm{vol} \%$ heat-inactivated FBS, $10 \mathrm{mmol} / \mathrm{L} \mathrm{N}$ (2-hydroxyethyl)piperazine-N'-(4-butanesulfonic acid), $\mathrm{pH}$ 7.4). Each drug and stimulant were premixed in the assay medium and incubated at $37^{\circ} \mathrm{C}$ for $30 \mathrm{~min}$. Then $0.05 \mathrm{~mL}$ of the mixture was placed into the lower chamber contained $0.45 \mathrm{~mL} /$ well of the assay medium. Then $0.2 \mathrm{~mL}$ of cell suspension $\left(6 \times 10^{5}\right.$ cells $)$ was added to the upper chamber and incubated for $3 \mathrm{~h}$ at $37^{\circ} \mathrm{C}$ in $5 \% \mathrm{CO}_{2}$. Cells passing through the membrane were collected from the lower well and counted with the EPICS XL/MCL flow cytometry system (Beckman Coulter, Fullerton, Calif, USA) followed by mixing of a predetermined number of flow-count fluorospheres (Beckman Coulter). The total number of cells that had migrated to the lower wells was determined by the ratio of cells to fluorospheres using the following formula: cells per microliter $=[($ cells counted $) /($ fluorospheres counted $)] \times$ fluorospheres/microliter. Each sample was assayed in triplicate.

\section{Statistical analysis}

Data were presented as means $\pm \mathrm{SE}$. The Student $t$ test following the F-test was used for analysis of differences between two groups. Multiple comparisons among treatment groups were assessed by one-way analysis of variance, followed by the Dunnett's test. Values of $P<.05$ were considered statistically significant. All statistical calculations were performed with the Statistical Analysis System (SAS Institute, Cary, NC, USA).

\section{RESULTS}

\section{Effects of olopatadine on the migration of THP-1 monocytes induced by S100A12 or RANTES}

The migration of THP-1 monocytes was studied in the presence of S100A12 or RANTES. S100A12 induced the 


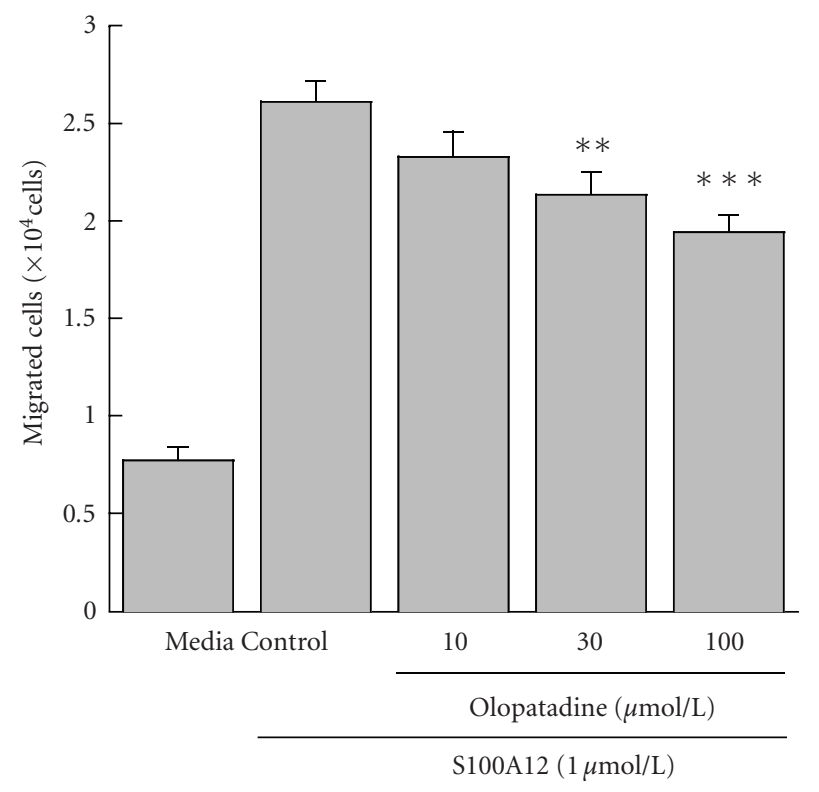

(a)

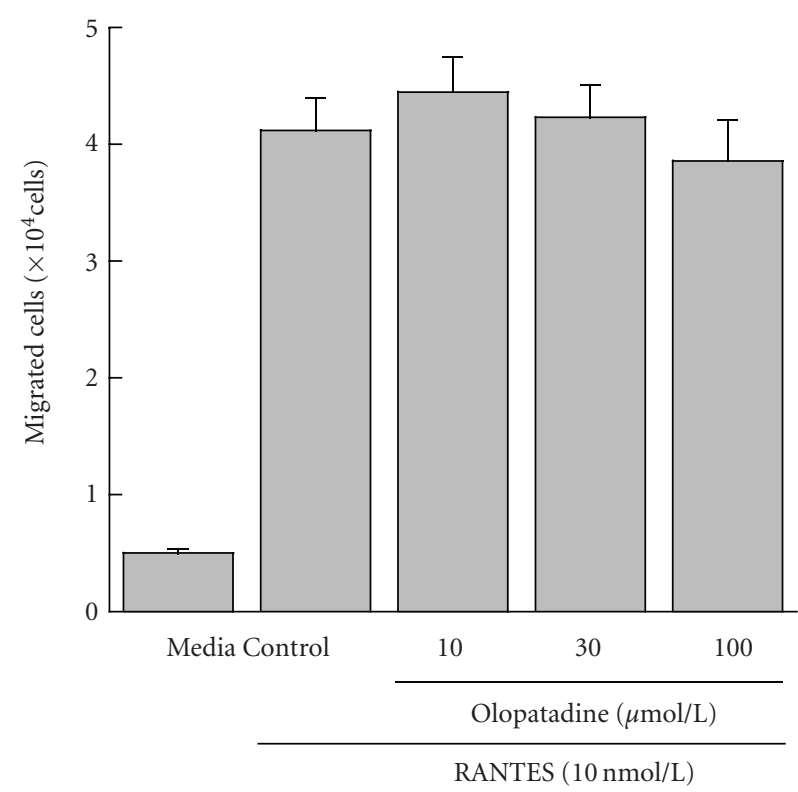

(b)

FIGURE 1: Effects of olopatadine on the migration of THP-1 cells induced by S100A12 (a) or RANTES (b). The numbers of cells migrating toward S100A12 $(1 \mu \mathrm{mol} / \mathrm{L})$ or RANTES $(10 \mathrm{nmol} / \mathrm{L})$ during $3 \mathrm{~h}$ incubation were determined by flow cytometry. The values in panel (a) are means \pm SE from 3 independent experiments $(n=9)$, and those in panel (b) are means \pm SE from 2 independent experiments $(n=6) .{ }^{* *} P<0.01 ;{ }^{* * *} P<0.001$ (versus control, Dunnett's test). Media, spontaneous migration.

migration of THP- 1 cells at concentrations between 0.75 and $2 \mu \mathrm{mol} / \mathrm{L}$ (data not shown). Therefore, we examined the effect of olopatadine on the activity of S100A12 at $1 \mu \mathrm{mol} / \mathrm{L}$, a submaximal concentration. After pretreatment of S100A12 for $30 \mathrm{~min}, 26095 \pm 1044$ cells/well migrated

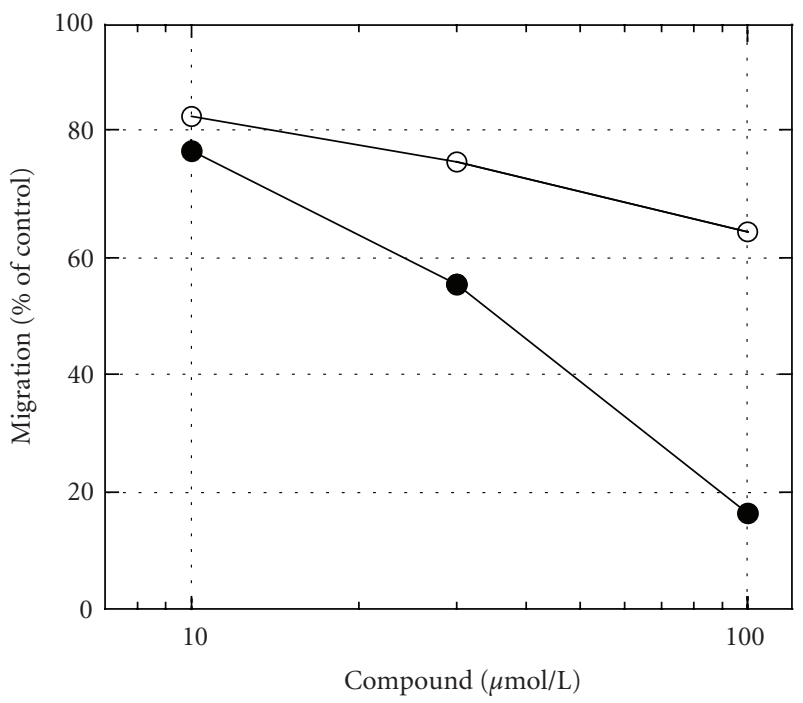

FIGURE 2: Comparison of the abilities of olopatadine (open circle) and amlexanox (closed circle) to inhibit THP-1 migration induced by S100A12. Migration assay was performed as described for the prior experiments. Values are percentages of control, and are the means of 2 independent experiments.

to the bottom chamber at $1 \mu \mathrm{mol} / \mathrm{L}$ of $\mathrm{S} 100 \mathrm{~A} 12$, indicating a 3.4-fold increase in comparison with the control value of $7699 \pm 693$ cells/well (Figure 1(a)). On the other hand, RANTES caused a 6.0-fold increase in the migration of THP1 cells at $10 \mathrm{nmol} / \mathrm{L}$ (Figure 1(b)). As shown in Figure 1(a), pretreatment of S100A12 with olopatadine resulted in a concentration-dependent reduction of THP- 1 cell migration induced by $S 100 \mathrm{~A} 12$, although the reduction was only partial. The reduction of THP- 1 cell migration by olopatadine is apparently not caused by inhibition of cell motility, since parallel studies showed that olopatadine had no effect on the migration of cells induced by RANTES (Figure 1(b)).

\section{Effect of amlexanox on THP-1 migration induced by S100A12}

Amlexanox is also reported to bind to S100A12 [12]. In addition, it has already been reported that amlexanox binds to $\mathrm{S} 100 \mathrm{~A} 13$ and represses the FGF-1 release induced by S100A13 [13, 14]. Accordingly, it is expected that amlexanox also inhibits the function of S100A12. Figure 2 shows that amlexanox suppressed THP-1 migration induced by S100A12 more efficiently than olopatadine. While amlexanox suppressed the THP-1 migration induced by RANTES to some extent (data not shown), it was weak in this respect compared with inhibition of migration by S100A12. It is likely that amlexanox suppresses the effect of S100A12 by binding to S100A12 rather than by inhibiting cell motility.

\section{Effects of ketotifen on the migration of THP-1 cells induced by S100A12 or RANTES}

Next, to determine whether histamine $\mathrm{H}_{1}$ receptor antagonistic activity of olopatadine is related inhibition of the 


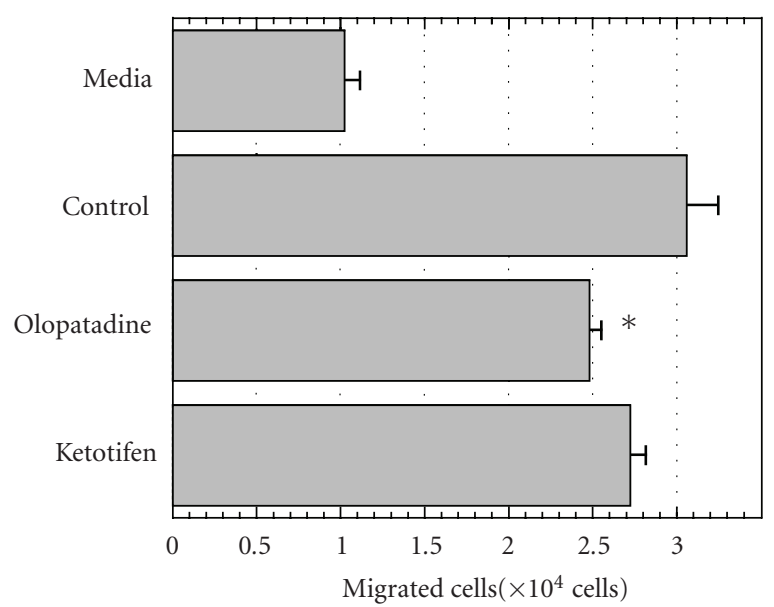

(a)

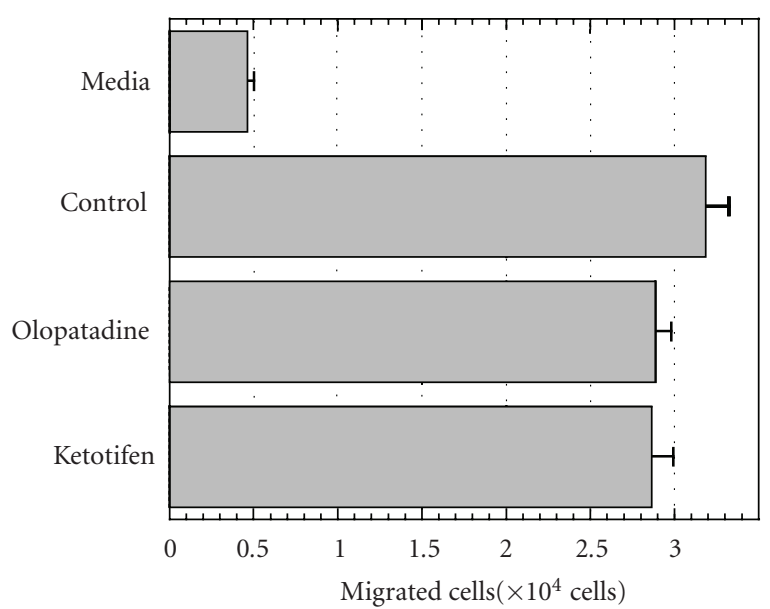

(b)

FIGURE 3: Effects of olopatadine or ketotifen on the migration of THP-1 cells induced by S100A12 (a) or RANTES (b). The numbers of cells migrating toward S100A12 $(1 \mu \mathrm{mol} / \mathrm{L})$ or RANTES $(10 \mathrm{nmol} / \mathrm{L})$ during $3 \mathrm{~h}$ incubation were counted. Values are means \pm SE from 2 independent experiments $(n=6) .{ }^{*} P<0.05$ (versus control, Student $t$ test). Media, spontaneous migration.

activity of S100A12, ketotifen, another histamine $\mathrm{H}_{1}$ receptor antagonist, was tested. Figure 3 shows that ketotifen $(50 \mu \mathrm{mol} / \mathrm{L})$ did not affect THP-1 migration induced by either S100A12 and RANTES, though it has already been shown that ketotifen has more potent histamine $\mathrm{H}_{1}$ receptor antagonistic activity than olopatadine $[21,22]$. These results suggest that olopatadine directly binds to S100A12 and inhibits its function rather than histamine $\mathrm{H}_{1}$ receptor antagonistic activity.

\section{DISCUSSION}

S100A12 has been identified as a binding protein to antiallergic drugs, such as olopatadine, amlexanox, and cromolyn, using drug-affinity columns [19]. S100A12 has multiple inflammatory effects [8-11]. If these drugs suppress the activity of S100A12, a new anti-inflammatory mechanism of action of these drugs can be demonstrated. In the present study, we confirmed that S100A12 induced the migration of THP-1, a human monocytic cell line, at $1 \mu \mathrm{mol} / \mathrm{L}$, and then that olopatadine and amlexanox suppressed this migration. Moreover, these drugs had little effect on the migration of THP- 1 cells induced by RANTES, suggesting that olopatadine and amlexanox bind to S100A12 specifically and suppress its activity.

Previous studies showed that S100A12 induced chemotactic responses at much lower concentrations (0.01$1 \mathrm{nmol} / \mathrm{L}$ ) $[9,10]$, whereas no response to $\mathrm{S} 100 \mathrm{~A} 12$ at lower concentrations was detected in our experiments. Recently, $\mathrm{x}$-ray analysis has revealed two crystal forms of recombinant S100A12 [21]. Therefore, the effective concentration of S100A12 may vary according to experimental conditions. It is possible that in some conditions lesser amounts of drugs are sufficient for suppression of the activity of. In fact, it is necessary to examine the effect of olopatadine on the function of S100A12 in vivo experiments.

Ketotifen in contrast to olopatadine did not suppress the THP-1 migration induced by S100A12, suggesting that olopatadine may have the distinctive feature of direct inhibition of the function of S100A12. This new mechanism of action of olopatadine may in addition to its potent histamine $\mathrm{H}_{1}$ receptor antagonistic activity provide high degree of efficacy in the treatment of allergic diseases.

\section{ACKNOWLEDGMENT}

We thank Dr Ryoji Kobayashi (Department of Signal Transduction Sciences, Kagawa University, Kagawa, Japan) for providing the human S100A12 cDNA.

\section{REFERENCES}

[1] Schafer BW, Heizmann CW. The S100 family of EF-hand calcium-binding proteins: functions and pathology. Trends in Biochemical Sciences. 1996;21(4):134-140.

[2] Hatakeyama T, Okada M, Shimamoto S, Kubota Y, Kobayashi R. Identification of intracellular target proteins of the calciumsignaling protein S100A12. European Journal of Biochemistry / FEBS. 2004;271(18):3765-3775.

[3] Steinbakk M, Naess-Andresen CF, Lingaas E, Dale I, Brandtzaeg P, Fagerhol MK. Antimicrobial actions of calcium binding leucocyte L1 protein, calprotectin. Lancet. 1990;336 (8718):763-765.

[4] Sohnle PG, Collins-Lech C, Wiessner JH. Antimicrobial activity of an abundant calcium-binding protein in the cytoplasm of human neutrophils. The Journal of Infectious Diseases. 1991;163(1):187-192.

[5] Brandtzaeg P, Gabrielsen TO, Dale I, Muller F, Steinbakk M, Fagerhol MK. The leucocyte protein L1 (calprotectin): a putative nonspecific defence factor at epithelial surfaces. Advances in Experimental Medicine and Biology. 1995;371A:201-206.

[6] Hessian PA, Fisher L. The heterodimeric complex of MRP-8 (S100A8) and MRP-14 (S100A9). Antibody recognition, epitope definition and the implications for structure. European Journal of Biochemistry / FEBS. 2001;268(2):353-363. 
[7] Devery JM, King NJ, Geczy CL. Acute inflammatory activity of the S100 protein CP-10. Activation of neutrophils in vivo and in vitro. The Journal of Immunology. 1994;152(4):1888-1897.

[8] Yang Z, Tao T, Raftery MJ, Youssef P, Di Girolamo N, Geczy CL. Proinflammatory properties of the human S100 protein S100A12. Journal of Leukocyte Biology. 2001;69(6):986-994.

[9] Hofmann MA, Drury S, Fu C, et al. RAGE mediates a novel proinflammatory axis: a central cell surface receptor for S100/calgranulin polypeptides. Cell. 1999;97(7):889-901.

[10] Miranda LP, Tao T, Jones A, et al. Total chemical synthesis and chemotactic activity of human S100A12 (EN-RAGE). FEBS Letters. 2001;488(1-2):85-90.

[11] Rouleau P, Vandal K, Ryckman C, et al. The calcium-binding protein S100A12 induces neutrophil adhesion, migration, and release from bone marrow in mouse at concentrations similar to those found in human inflammatory arthritis. Clinical Immunology. 2003;107(1):46-54.

[12] Shishibori T, Oyama Y, Matsushita O, et al. Three distinct anti-allergic drugs, amlexanox, cromolyn and tranilast, bind to S100A12 and S100A13 of the S100 protein family. Biochemical Journal. 1999;338(pt 3):583-589.

[13] Mouta Carreira C, LaVallee TM, Tarantini F, et al. S100A13 is involved in the regulation of fibroblast growth factor-1 and p40 synaptotagmin-1 release in vitro. The Journal of Biological Chemistry. 1998;273(35):22224-22231.

[14] Prudovsky I, Bagala C, Tarantini F, et al. The intracellular translocation of the components of the fibroblast growth factor 1 release complex precedes their assembly prior to export. The Journal of Cell Biology. 2002;158(2):201-208.

[15] Ohmori K, Hayashi K, Kaise T, et al. Pharmacological, pharmacokinetic and clinical properties of olopatadine hydrochloride, a new antiallergic drug. The Japanese Journal of Pharmacology. 2002;88(4):379-397.

[16] Hayashi K, Kaise T, Ohmori K, Ishii A, Karasawa A. Effects of olopatadine hydrochloride on the cutaneous vascular hyperpermeability and the scratching behavior induced by poly-Larginine in rats. The Japanese Journal of Pharmacology. 2001;87 (2):167-170.

[17] Fukuishi N, Matsuhisa M, Shimono T, et al. Inhibitory effect of olopatadine on antigen-induced eosinophil infiltration and the LFA-1 and Mac-1 expression in eosinophils. The Japanese Journal of Pharmacology. 2002;88(4):463-466.

[18] Tamura T, Matsubara M, Takada C, et al. Effects of olopatadine hydrochloride, an antihistamine drug, on skin inflammation induced by repeated topical application of oxazolone in mice. British Journal of Dermatology. 2004;151(6):1133-1142.

[19] Okada M, Tokumitsu H, Kubota Y, Kobayashi R. Interaction of S100 proteins with the antiallergic drugs, olopatadine, amlexanox, and cromolyn: identification of putative drug binding sites on S100A1 protein. Biochemical and Biophysical Research Communications. 2002;292(4):1023-1030.

[20] Yamashita K, Oyama Y, Shishibori T, Matsushita O, Okabe A, Kobayashi R. Purification of bovine S100A12 from recombinant Escherichia coli. Protein Expression and Purification. 1999;16(1):47-52.

[21] Nonaka H, Ishii A, Miki I, Ichimura M, Kase H. Affinity of KW-4679, a novel antiallergic drug, for various receptors and ex vivo histamine $\mathrm{H} 1$ receptors. Clinical pharmacology \& Therapeutics. 1995;5(10):1817-1824. (in Japanese).

[22] Moroz OV, Dodson GG, Wilson KS, Lukanidin E, Bronstein IB. Multiple structural states of S100A12: A key to its functional diversity. Microscopy Research and Technique. 2003;60(6):581-592. 


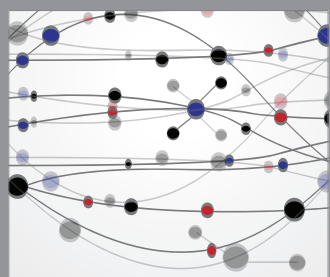

The Scientific World Journal
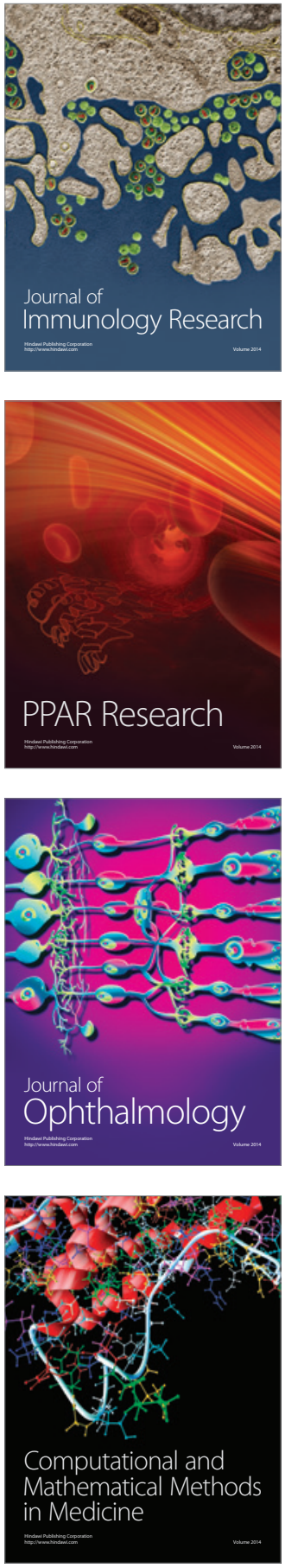

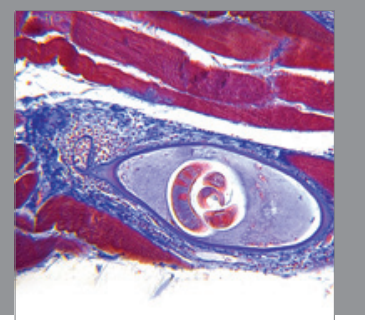

Gastroenterology

Research and Practice
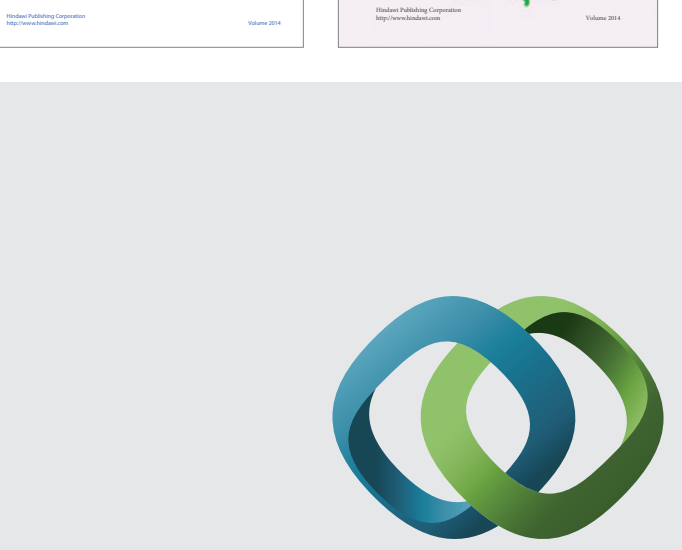

\section{Hindawi}

Submit your manuscripts at

http://www.hindawi.com
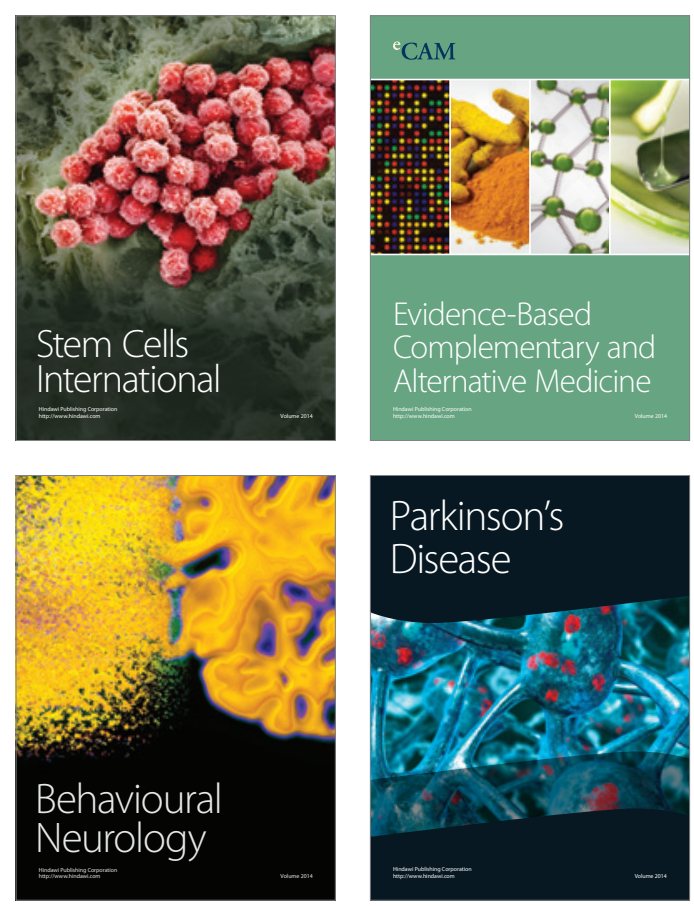

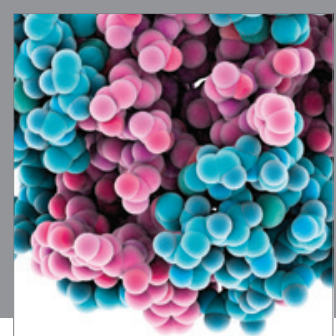

Journal of
Diabetes Research

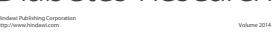

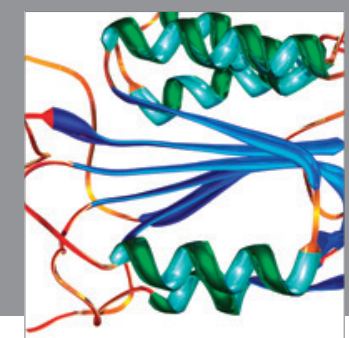

Disease Markers
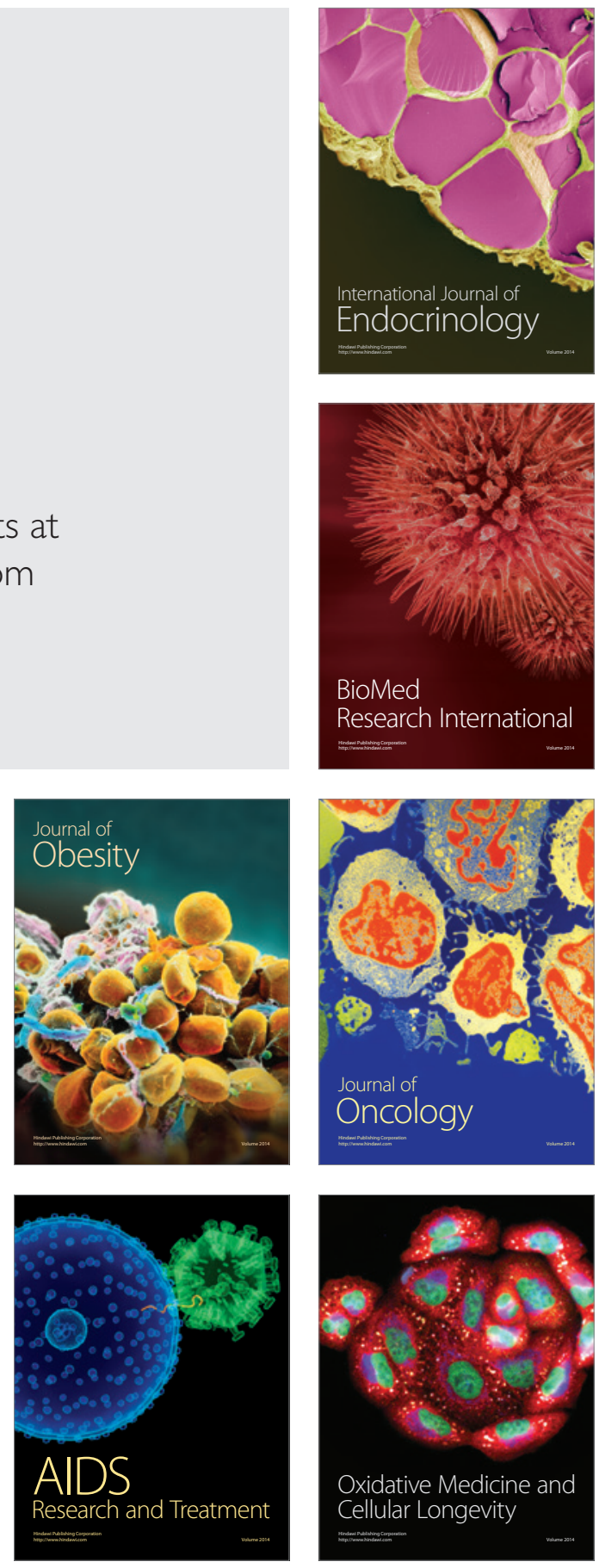